\title{
Integrated analysis of long non-coding RNA-associated ceRNA network reveals potential IncRNA biomarkers in human lung adenocarcinoma
}

\author{
JING SUI*, YUN-HUI LI*, YAN-QIU ZHANG, CHENG-YUN LI, XIAN SHEN, WEN-ZHUO YAO, \\ HUI PENG, WEI-WEI HONG, LI-HONG YIN, YUE-PU PU and GE-YU LIANG
}

Key Laboratory of Environmental Medicine Engineering, Ministry of Education, School of Public Health, Southeast University, Nanjing, Jiangsu 210009, P.R. China

Received August 4, 2016; Accepted September 27, 2016

DOI: $10.3892 /$ ijo.2016.3716

\begin{abstract}
Accumulating evidence has highlighted the important roles of long non-coding RNAs (lncRNAs) acting as competing endogenous RNAs (ceRNAs) in tumor biology. However, the roles of cancer specific lncRNAs in lncRNArelated ceRNA network of lung adenocarcinoma (LUAD) are still unclear. In the present study, the 465 RNA sequencing profiles in LUAD patients were obtained from The Cancer Genome Atlas (TCGA) database, which provides large sample RNA sequencing data free of charge, and 41 cancer specific lncRNAs, 25 miRNAs and 1053 mRNAs (fold change $>2$, $\mathrm{P}<0.05)$ were identified. Then, the IncRNA-miRNA-mRNA ceRNA network of LUAD was constructed with 29 key lncRNAs, 24 miRNAs and 72 mRNAs. Subsequently, we selected these 29 key lncRNAs to analyze their correlation with clinical features, and 21 of them were aberrantly expressed with tumor pathological stage, TNM staging system, lymph node metastasis and patient outcome assessment, respectively. Furthermore, there were 5 lncRNAs (BCRP3, LINC00472, CHIAP2, BMS1P20 and UNQ6494) positively correlated with overall survival (OS, log-rank $\mathrm{P}<0.05)$. Finally, 7 cancer specific lncRNAs were randomly selected to verify the expression in 53 newly diagnosed LUAD patients using qRT-PCR. The expression results between TCGA and qRT-PCR were $100 \%$ in agreement. The correlation between AFAP1-AS1 and LINC00472 and clinical features were also confirmed. Thus, our results showed the lncRNA expression profiles and we constructed an IncRNA-miRNA-mRNA ceRNA network
\end{abstract}

Correspondence to: Professor Ge-Yu Liang, Key Laboratory of Environmental Medicine Engineering, Ministry of Education, School of Public Health, Southeast University, Nanjing, Jiangsu 210009, P.R. China

E-mail: lianggeyu@163.com

*Contributed equally

Key words: long non-coding RNAs, lung adenocarcinoma, The Cancer Genome Atlas, competing endogenous RNAs network, competing endogenous RNAs, overall survival in LUAD. The present study provides novel insight for better understanding of IncRNA-related ceRNA network in LUAD and facilitates the identification of potential biomarkers for diagnosis and prognosis.

\section{Introduction}

Lung cancer is the most commonly diagnosed and fatal cancer, which was estimated as 1.8 million new cases and almost 1.6 million deaths occurring worldwide in $2012(1,2)$. Lung adenocarcinoma (LUAD) is major pathologic subtype of lung cancer in non-smoking males, and in females (smokers or non-smokers) $(3,4)$. Based on the GLOBOCAN series, the incidence of LUAD has significantly increased over the past few decades in Africa, America and Asia (5). LUAD mainly occurs in the peripheral region of the lungs, with ability to metastasize to the lymph nodes and other organs. Previous studies have shown that several genetic abnormalities were associated with the initiation and development of LUAD (6-10), but the pathogenesis contributing to biological properties of LUAD remain inconclusive.

Non-coding RNAs (ncRNAs) are a class of RNA molecules with no protein-coding function, which are widely expressed in organisms. ncRNAs are divided into several subtypes, such as microRNAs (miRNAs), small nucleolar RNA (snoRNA), small nuclear RNA (snRNA), ribosomal RNA (rRNA), transfer ribonucleic acidtRNA (tRNA) and long non-coding RNA (lncRNA).

Recent studies indicate that ncRNAs play important biological roles in various biological processes, such as regulate gene expression, cell proliferation, differentiation and apoptosis (11-15). lncRNAs, ranging from 200 nucleotides to $100 \mathrm{~kb}$ in length, have become an area of increased research $(12,16)$. IncRNAs may regulate gene expression at the transcriptional, post-transcriptional and epigenetic levels (17-19). Because of the stronger tissue specificity, lncRNAs can be more effective as early diagnosis and screening of cancer biomarkers (20). Increasing number of studies on lncRNAs has been performed in various types of cancer, such as lung, esophageal, gastric, hepatocellular and colorectal cancer (21-25.) The aberrantly expressed lncRNAs, which 
regulate initiation, development, invasion and metastasis of tumors have been detected in LUAD $(10,26)$.

The ceRNA (competing endogenous RNA) hypothesis was proposed as a novel regulatory mechanism between non-coding RNA and coding RNA (27). IncRNA can interact with the miRNA by miRNA-binding sites (MREs), thereby regulating gene expression (28). The complex crosstalk of ceRNA network has been detected in many different diseases (19). Zhang et al (29) analyzed the lncRNA profiles in 372 hepatocellular cell carcinoma (HCC) patients from The Cancer Genome Atlas (TCGA) (http://cancergenome.nih. gov/) and NCBI GEO Omnibus (GSE65485), and constructed an IncRNA-miRNA-mRNA network in HCC. Based on the ceRNA hypothesis, Li et al (30) selected 361 RNA sequencing profiles of gastric cancer (GC) patients from TCGA, and further analysis of the associations between these RNA data and related patient clinical information. Then, the lncRNAmiRNA-mRNA ceRNA network of GC was constructed.

A recent study showed the differential expression of lncRNA profile of LUAD using high-throughput microarray analysis from 33 LUAD patients (31). However, studies with large scale samples and microarray detection is still rare, and the relationship between cancer specific lncRNAs and clinical features are unclear. TCGA database is a public platform, in which sequencing data of lncRNA, miRNA and mRNA for LUAD can be downloaded. To improve the reliability and accuracy of the studies, we detected lncRNAs in LUAD using data sets from TCGA database.

In the present study, the RNA sequencing data of 521 LUAD samples and 49 adjacent non-tumor lung tissues samples were collected from TCGA database. This is the first study to investigate the cancer specific lncRNA from large scale sequencing database (TCGA) and ceRNA network constructed in LUAD. Quantificational real-time polymerase chain reaction (qRTPCR) was used to verify the bioinformatic analysis results in LUAD tumor tissues and adjacent non-tumor lung tissues from 53 newly diagnosed LUAD patients. The present study can help elaborate the function of IncRNAs through lncRNAmiRNA-mRNA ceRNA network in LUAD.

\section{Materials and methods}

Patients and samples. This study was approved by the ethics committee of the Zhongda Hospital Southeast University. A total of 521 LUAD cases were obtained from the TCGA database. The exclusion criteria were set as follows: i) histologic diagnosis is not LUAD; ii) suffering of other malignancy except LUAD; iii) patients samples without complete data for analysis; and iv) overall survival (OS) $>5$ years. Overall, a total of 465 LUAD patients were included in this study. lncRNA expression profiles for normal lung tissue samples were obtained from adjacent non-tumor lung tissues $(n=49)$. In addition, there are 170 LUAD patients with lymph node metastasis and 295 LUAD patients with non-lymph node metastasis. According to the Union for International Cancer Control (UICC), well and moderately-differentiated LUAD (stage I-II) were 359 patients, poorly-differentiated LUAD (stage III-IV) were 106 patients. Both RNA expression data and clinical data, including staging information and outcome of LUAD patients downloaded from the TCGA Data Portal, are open-access and publicly available. The present study meets the publication guidelines provided by TCGA (http:// cancergenome.nih.gov/publications/publicationguidelines).

In addition, 53 frozen tissue specimens (tumor tissues and their paired adjacent non-cancerous tissues) from LUAD patients collected between 2006 and 2015 were obtained from Nanjing Chest Hospital Medical School of Southeast University. Tissues were snap-frozen in RNAlater (Ambion, Austin, TX, USA) and stored in liquid nitrogen immediately after surgical resection until total RNA extraction and analysis. Samples were received with a pathology report and a quality assessment report verifying collection of tumor and/or adjacent non-tumor lung tissues. Informed consent forms were obtained from all patients included in the present study.

RNA sequence data procession and computational analysis. The LUAD RNA expression data (level 3) of the corresponding patients were downloaded from the TCGA Data Portal (March 2016). The RNA sequencing raw reads (lncRNA and mRNA) were post-processed and normalized by TCGA RNASeqV2 system. Level 3, normalized miRNA expression data (the calculated expression for all reads aligning to a particular miRNA per sample) were downloaded from the TCGA Data Portal performed using Illumina HiSeq and Illumina GA microRNA sequencing platforms (Illumina, Inc., Hayward, CA, USA) and quantile normalized before performing analysis. No further normalizations were applied to the lncRNA, miRNA and mRNA expression profile data in level 3, because these data were already normalized by TCGA. To detect the differential expression of lncRNA, miRNA and mRNA, samples were divided into LUAD patient tumor tissues vs. adjacent nontumor lung tissues, lymph node metastasis of LUAD patients vs. non-lymph node metastasis of LUAD patients, well and moderately-differentiated LUAD (stages I-II) vs. poorlydifferentiated LUAD (stages III-IV), respectively. For further analysis, intersection of IncRNA, miRNA and mRNA was selected. The flow chart for bioinformatics analysis is depicted in Fig. 1.

Functional enrichment analysis. To understand the underlying biological processes and pathways of aberrantly expressed intersection genes, we use the DAVID (Database for Annotation, Visualization, and Integrated Discovery) Bioinformatics resources (http://david.abcc.ncifcrf.gov/) (32), to conduct functional enrichment analysis, and we were only interested in GO (Gene Ontology) biological processes and KEGG (Kyoto Encyclopedia of Genes and Genomes) pathways at the significant level $(\mathrm{P}<0.05$ and an enrichment score of $>1.5$ ).

Construction of IncRNA-miRNA-mRNA ceRNA network. The lncRNA-miRNA-mRNA ceRNA network was constructed based on the relationship among lncRNA, miRNA and mRNA. It is established that the post-transcriptional regulation of mRNA transcripts could be bound by single-stranded miRNAs. In the present study, the IncRNA-miRNA-mRNA ceRNA network, which was based on the theory that lncRNAs can have a direct interaction by invoking the miRNA sponge to regulate the activity of mRNAs (33). To construct ceRNA network, the human miRNA-mRNA and miRNA-lncRNA 


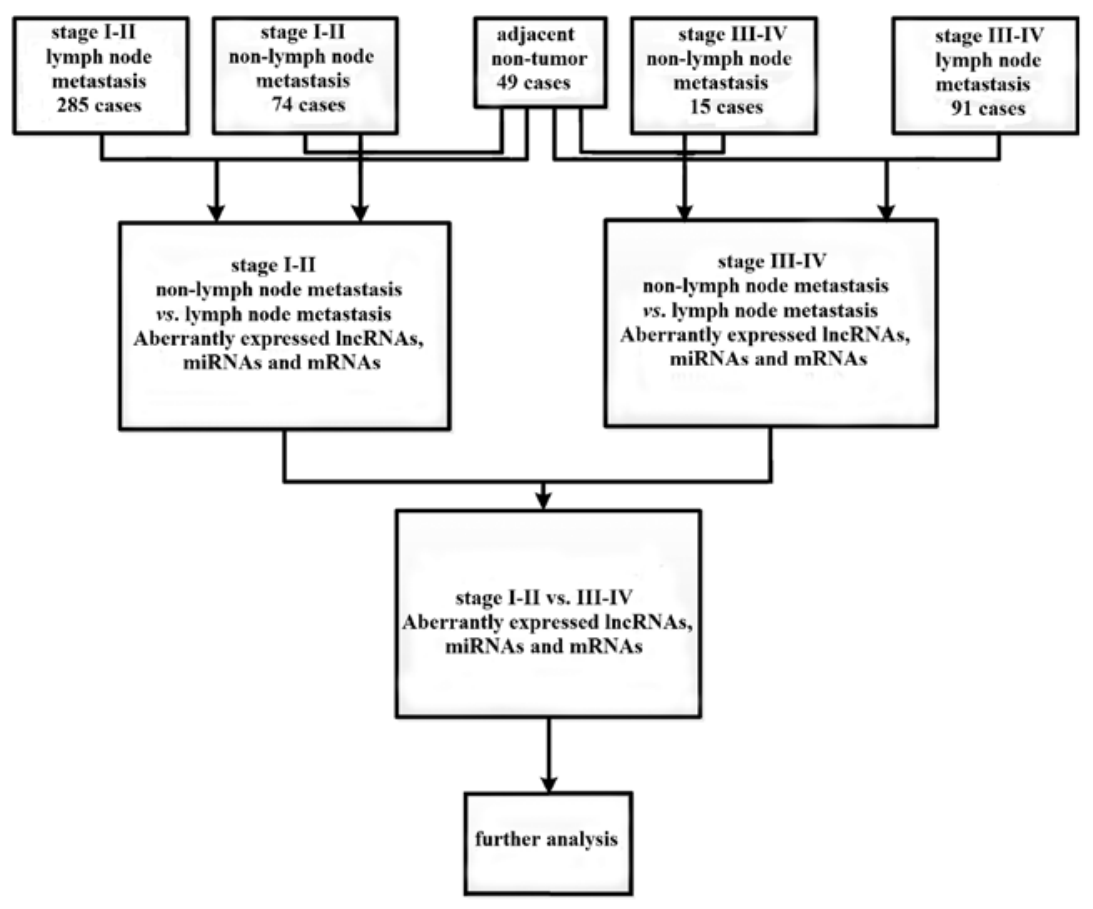

Figure 1. Flow chart of bioinformatics analysis.

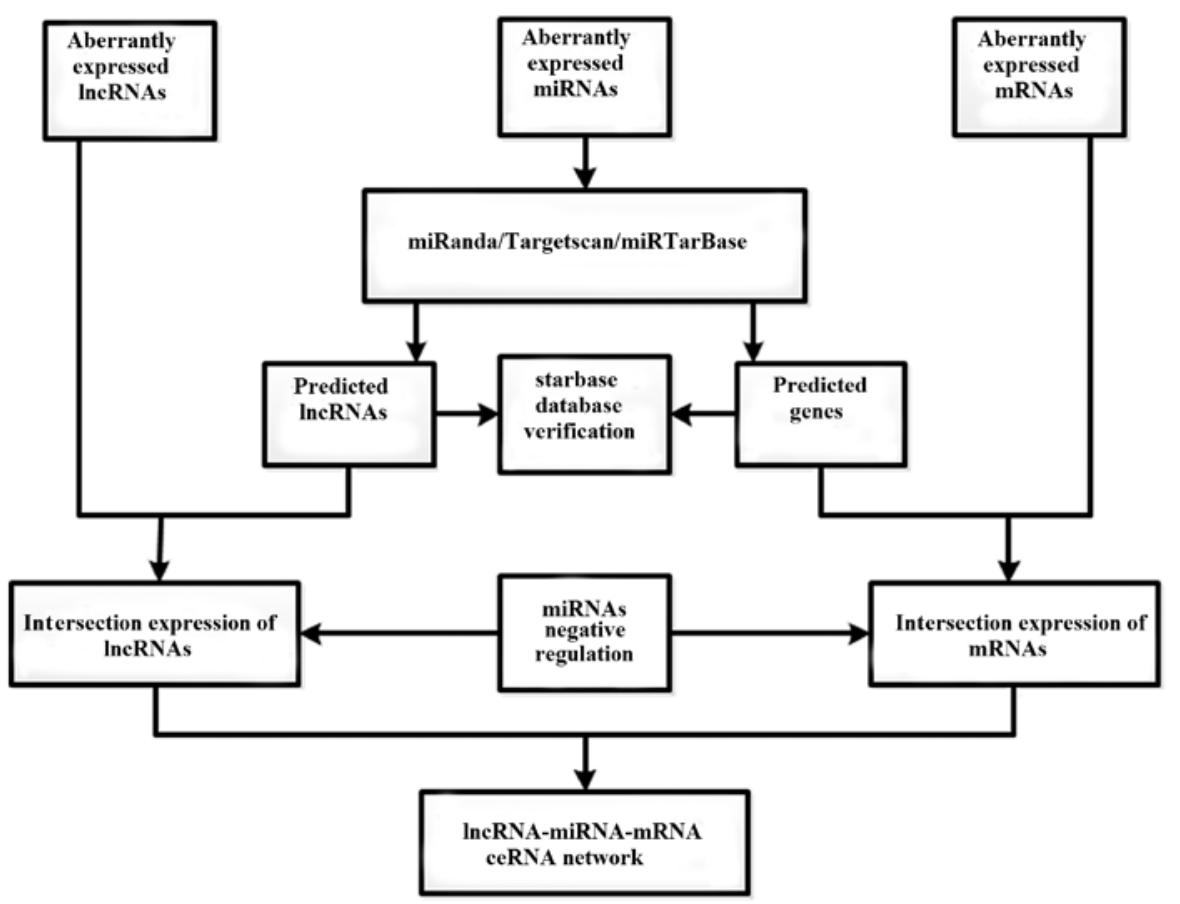

Figure 2. The flow chart of ceRNA network analysis.

interactions could be downloaded from the starBase V2.0 database (http://starbase.sysu.edu.cn/) (34), then aberrantly expressed intersection IncRNA, miRNA and mRNA with fold change $>2.0$ and/or fold change $<0.5$ and $\mathrm{P}<0.05$ were retained. The miRanda tools (http://www.microrna.org/microrna/home. do) were used to predict the lncRNA-miRNA interactions. Targetscan (http://www.targetscan.org/) and miRTarBase (http://mirtarbase.mbc.nctu.edu.tw/) (35) were performed to predict the mRNAs targeted by miRNAs. The results could be verified in starBase database. Furthermore, in the present study the predicted miRNAs and aberrantly expressed data of TCGA were combined to select the intersection lncRNAs and mRNAs. To construct ceRNA network, the miRNAs negatively regulated intersection expression of lncRNAs and mRNAs were selected, based on the theory of ceRNA. Cytoscape v3.0 (30) was performed to construct and visualize the IncRNA-miRNA-mRNA ceRNA network. The flow chart for ceRNA network construction is described in Fig. 2. 

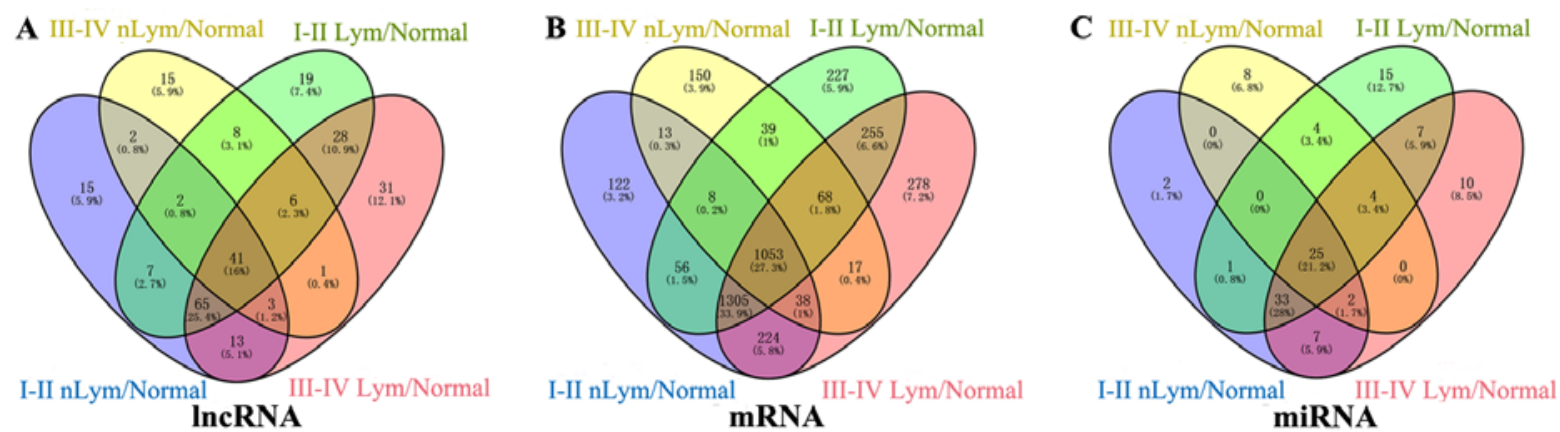

Figure 3. Venn diagram analysis of aberrantly expressed (A) lncRNAs, (B) mRNAs and (C) miRNAs between I-II Lym/Normal, I-II nLym/Normal, III-IV Lym/Normal and III-IV nLym/Normal. Lym, lymph node metastasis; nLym, non-lymph node metastasis; normal represent adjacent non-tumor lung tissues.

The correlations between LUAD specific lncRNAs and clinical features. According to the bioinformatics analysis and the ceRNA network, specific lncRNAs were selected. We further analyzed the clinical features including gender, tumor pathological stage, TNM staging system, lymph node metastasis and patient outcome assessment. Subsequently, to identify the specific IncRNAs with prognostic characteristics, the univariate Cox proportional hazards regression model was used to analyze the association between the specific lncRNAs and the LUAD patient survival time.

Total RNA extraction and qRT-PCR verification. We randomly selected 7 of the specific lncRNAs in lncRNA-miRNA-mRNA ceRNA network, and validated the reliability and validity of the results in 53 newly diagnosed LUAD patients using qRT-PCR.

The total RNA was isolated with TRIzol reagent (Invitrogen, Carlsbad, CA, USA) according to the manufacture's protocol, and RNA purity was detected by NanoDrop 2000 spectrometer (Thermo Fisher Scientific, Waltham, MA, USA). Reverse transcription reactions using A3500 reverse transcription system kit (Promega, Madison, WI, USA) was conducted in two steps according to the manufacturer's protocol. QRT-PCR was carried out to detect the expression levels of candidate lncRNAs with the StepOnePlus Real-Time PCR system (Applied Biosystems, Foster City, CA, USA). QRT-PCR was then performed using A6001 GoTaq qPCR Master Mix (Promega) according to the manufacturer's protocol. All primers were purchased from Generay Biotech Co., Ltd. (Shanghai, China). The Ct-value for each sample was calculated with the $\Delta \Delta \mathrm{Ct}$ method (10), and fold change results were presented as $2^{-\Delta \Delta \mathrm{Ct}}$, where $\Delta \Delta \mathrm{Ct}=\left(\mathrm{Ct}_{\mathrm{RNAs}}-\mathrm{Ct}_{\mathrm{GAPDH}}\right)$ tumor - $\left(\mathrm{Ct}_{\mathrm{RNAs}}-\mathrm{Ct}_{\mathrm{GAPDH}}\right)_{\text {adjacent non-tumor tissues }}$.

Statistical analysis. All data are presented as mean \pm SD, and statistically compared performing the paired t-test. The significance level was set as 0.001 as default to control the false discovery rate (FDR). Values of $\mathrm{P}<0.05$ were considered statistically significant. The statistical analyses were performed by the SPSS 21.0.

\section{Results}

LUAD specific lncRNAs in LUAD patients. We identified a total of $1806 \mathrm{lncRNAs}$ from TCGA database. Analysis of IncRNA expression profiles in LUAD patient tissues $(n=465)$ compared with adjacent non-tumor lung tissues $(n=49)$ identified 256 aberrantly expressed lncRNAs (absolute fold change $>2, \mathrm{P}<0.01)$. Of these, $103 \mathrm{lncRNAs}$ were downregulated and 153 lncRNAs were upregulated. Based on these data, further analysis was performed between tumor stage (lymph node metastasis) LUAD patient tissues and adjacent non-tumor lung tissues. A total of 148 aberrantly expressed lncRNAs were selected between stages I-II (non-lymph node metastasis) LUAD patient tissues and adjacent non-tumor lung tissues, 176 aberrantly expressed lncRNAs were selected between stages III-IV (non-lymph node metastasis) LUAD patient tissues and adjacent non-tumor lung tissues, 78 aberrantly expressed IncRNAs were selected between stages I-II (lymph node metastasis) LUAD patient tissues and adjacent non-tumor lung tissues, 188 aberrantly expressed lncRNAs were selected between stages III-IV (lymph node metastasis) LUAD patient tissues and adjacent non-tumor lung tissues. To further enhance the data reliability, we selected 41 aberrantly expressed lncRNAs (17 downregulated and 24 upregulated) in the intersection of the above 4 groups to build the IncRNAmiRNA-mRNA ceRNA network (Fig. 3A and Table I).

Functional enrichment analysis. In order to understand the functions of aberrantly expressed genes in this study, the intersection mRNAs were analyzed by DAVID bioinformatics resources. In total, we identified 18633 mRNAs from TCGA database. A total of 3853 aberrantly expressed mRNAs were found between LUAD patient tissues and adjacent non-tumor lung tissues (absolute fold change $>2, \mathrm{P}<0.01$ ). Based on these data, aberrantly expressed genes were further analyzed; 2819 and 1386 aberrantly expressed mRNAs were respectively identified between stages I-II (non-lymph/lymph node metastasis) LUAD patient tissues and adjacent non-tumor lung tissues, 3011 and 3238 aberrantly expressed mRNAs were, respectively, identified between stages III-IV (non-lymph/lymph node metastasis) LUAD patient tissues and adjacent non-tumor lung tissues. Subsequently, 1053 mRNAs were selected from the intersection of the above 4 groups, and divided into downregulated and upregulated genes for further functional analysis (Fig. 3B).

It revealed enrichment of $668 \mathrm{GO}$ categories and 87 KEGG categories (P-value of $<0.05$ and an enrichment score of $>1.5$; Figs. 4 and 5). It was found that the top GO biological process of downregulated and upregulated genes was G-protein coupled receptor signaling pathway (GO:0007186) and mitotic cell cycle (GO:0000278) (Fig. 4), respectively. The 
Table I. Aberrantly expressed intersection lncRNAs between I-II Lym/Normal, I-II nLym/Normal, III-IV Lym/Normal and III-IV $\mathrm{nLym} /$ Normal $^{\mathrm{a}, \mathrm{b}, \mathrm{c}}$.

\begin{tabular}{|c|c|c|c|c|c|c|}
\hline lncRNAs & Gene ID & Chromosome & Regulation & Fold-change & $-\log (p)$ & $-\log \left(\mathrm{FDR}^{\mathrm{d}}\right)$ \\
\hline WWC2-AS2 & 152641 & 4 & Downregulation & $-3.306 \pm 0.220$ & 6.757 & 5.243 \\
\hline LINC00472 & 79940 & 6 & Downregulation & $-7.018 \pm 0.423$ & 7.000 & 6.490 \\
\hline AGPAT4-IT1 & 79992 & 6 & Downregulation & $-3.200 \pm 0.086$ & 3.916 & 2.406 \\
\hline CES1P1 & 51716 & 16 & Downregulation & $-7.407 \pm 2.079$ & 6.240 & 4.583 \\
\hline FAM95B1 & 100133036 & 9 & Downregulation & $-2.581 \pm 0.390$ & 3.334 & 1.950 \\
\hline LINC00930 & 100144604 & 15 & Downregulation & $-4.124 \pm 1.099$ & 5.140 & 3.480 \\
\hline RAMP2-AS1 & 100190938 & 17 & Downregulation & $-4.819 \pm 0.520$ & 6.903 & 5.513 \\
\hline BRE-AS1 & 100302650 & 2 & Downregulation & $-3.922 \pm 1.397$ & 4.080 & 2.534 \\
\hline CHIAP2 & 149620 & 1 & Downregulation & $-43.011 \pm 6.456$ & 6.903 & 5.513 \\
\hline LINC01105 & 150622 & 2 & Downregulation & $-5.797 \pm 1.526$ & 3.721 & 2.254 \\
\hline LINC00961 & 158376 & 9 & Downregulation & $-6.061 \pm 0.683$ & 7.000 & 5.774 \\
\hline LINC00908 & 284276 & 18 & Downregulation & $-3.810 \pm 0.695$ & 4.337 & 2.748 \\
\hline BCRP3 & 644165 & 22 & Downregulation & $-2.759 \pm 0.328$ & 4.190 & 2.625 \\
\hline MGC27382 & 149047 & 1 & Downregulation & $-11.799 \pm 0.396$ & 7.000 & 6.490 \\
\hline RPL13AP17 & 399670 & 7 & Downregulation & $-47.619 \pm 10.808$ & 7.000 & 6.490 \\
\hline SIGLEC16 & 400709 & 19 & Downregulation & $-3.478 \pm 0.259$ & 3.413 & 2.023 \\
\hline TPTEP1 & 387590 & 22 & Downregulation & $-3.200 \pm 1.353$ & 4.274 & 2.525 \\
\hline C1orf220 & 400798 & 1 & Upregulation & $4.635 \pm 0.649$ & 5.078 & 3.409 \\
\hline LINC00319 & 284836 & 21 & Upregulation & $6.750 \pm 1.475$ & 4.638 & 3.027 \\
\hline LINC00471 & 151477 & 2 & Upregulation & $2.423 \pm 0.135$ & 3.423 & 2.033 \\
\hline ZNF252P-AS1 & 286103 & 8 & Upregulation & $3.685 \pm 0.681$ & 3.623 & 2.190 \\
\hline DDX12P & 440081 & 12 & Upregulation & $4.395 \pm 1.037$ & 4.232 & 2.683 \\
\hline DGCR5 & 26220 & 22 & Upregulation & $9.073 \pm 2.637$ & 5.049 & 3.384 \\
\hline FER1L4 & 80307 & 20 & Upregulation & $21.753 \pm 9.600$ & 5.199 & 3.515 \\
\hline ANKRD36BP2 & 645784 & 2 & Upregulation & $5.205 \pm 1.082$ & 3.520 & 2.109 \\
\hline LOC 100132111 & 100132111 & 1 & Upregulation & $3.048 \pm 0.504$ & 3.361 & 1.984 \\
\hline LOC100133985 & 100133985 & 2 & Upregulation & $2.888 \pm 0.318$ & 3.471 & 2.069 \\
\hline LINC00896 & 150197 & 22 & Upregulation & $5.338 \pm 0.378$ & 7.000 & 6.245 \\
\hline LOC285629 & 285629 & 5 & Upregulation & $9.730 \pm 5.681$ & 7.000 & 6.245 \\
\hline LRRC37A6P & 387646 & 10 & Upregulation & $2.860 \pm 0.538$ & 3.322 & 1.954 \\
\hline LOC399815 & 399815 & 10 & Upregulation & $8.038 \pm 1.458$ & 7.000 & 6.245 \\
\hline AFAP1-AS1 & 84740 & 4 & Upregulation & $46.210 \pm 7.498$ & 7.000 & 6.245 \\
\hline BMS1P20 & 96610 & 22 & Upregulation & $4.468 \pm 0.767$ & 3.550 & 2.132 \\
\hline PVT1 & 5820 & 8 & Upregulation & $5.335 \pm 1.037$ & 5.949 & 4.199 \\
\hline RAET1K & 646024 & 6 & Upregulation & $3.463 \pm 0.161$ & 7.000 & 5.568 \\
\hline SNHG4 & 724102 & 5 & Upregulation & $3.625 \pm 0.117$ & 5.505 & 3.791 \\
\hline UCA1 & 652995 & 19 & Upregulation & $5.445 \pm 0.844$ & 6.456 & 4.695 \\
\hline TCAM1P & 146771 & 17 & Upregulation & $4.053 \pm 0.409$ & 4.205 & 2.661 \\
\hline CECR7 & 100130418 & 22 & Upregulation & $4.055 \pm 0.821$ & 4.428 & 2.850 \\
\hline ALOX12P2 & 245 & 17 & Upregulation & $3.623 \pm 0.261$ & 3.787 & 2.321 \\
\hline UNQ6494 & 100129066 & 9 & Upregulation & $2.330 \pm 0.881$ & 3.549 & 1.940 \\
\hline
\end{tabular}

a41 LUAD specific lncRNAs for ceRNA network construction with absolute fold change $>2.0, \mathrm{P}<0.05$. ${ }^{\mathrm{b}} \mathrm{I}$, II, III and IV, TNM stage I, II, III and IV. ' $\mathrm{Lym}$, lymph node metastasis; nLM, non-lymph node metastasis; normal represents adjacent non-tumor lung tissues; ${ }^{\mathrm{d} F D R}$, the false discovery rate of the lncRNAs, using Benjamini and Hochberg (1995) method.

top 15 KEGG pathways of downregulated and upregulated genes are also described in Fig. 5. Of these above pathways, PI3K-Akt signaling pathway, p53 signaling pathway, MAPK signaling pathway and Wnt signaling pathway are involved in development, invasion and metastases of cancer. In addition, there were other cancer-related pathways including pathways in cancer, small cell lung, pancreatic and bladder cancer and glioma. 

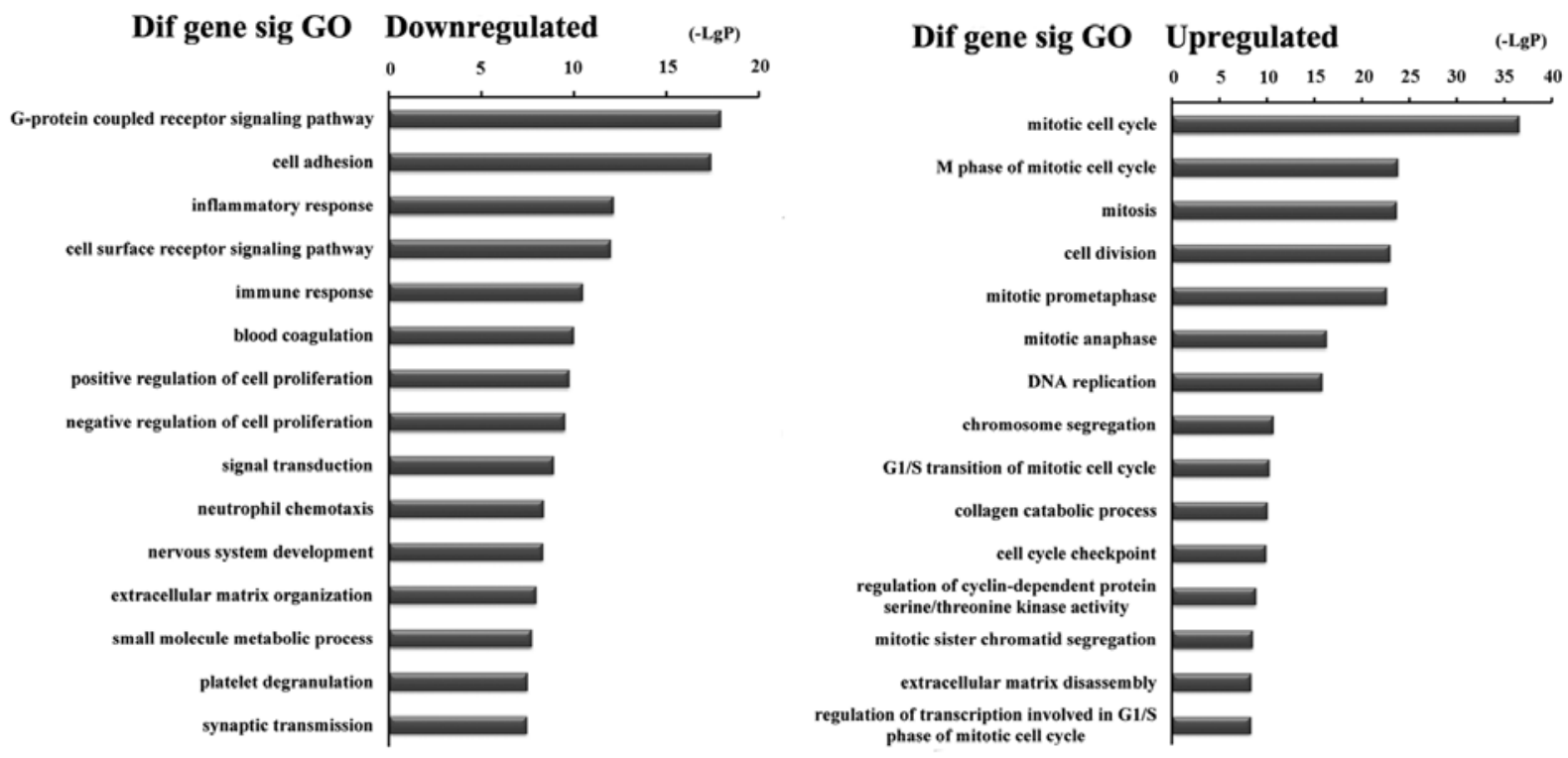

Figure 4. Top 15 enrichment of GO terms for aberrantly expressed intersection mRNAs (the bar plot shows the enrichment scores of the significant top 15 enrichment GO terms).

Table II. miRNAs that may target LUAD specific IncRNAs.

\begin{tabular}{|c|c|}
\hline $\operatorname{lncRNAs}$ & miRNAs \\
\hline LINC00961 & hsa-miR-127-5p, hsa-miR-96-5p \\
\hline SIGLEC16 & hsa-miR-127-5p \\
\hline ALOX12P2 & hsa-miR-133a-3p, hsa-miR-30a-3p, hsa-miR-486-5p \\
\hline BCRP3 & hsa-miR-135b-5p \\
\hline LINC01105 & hsa-miR-135b-5p, hsa-miR-143-5p, hsa-miR-182-5p, hsa-miR-708-5p, hsa-miR-96-5p \\
\hline FER1L4 & hsa-miR-139-3p, hsa-miR-30c-2-3p \\
\hline LINC00319 & hsa-miR-139-3p \\
\hline LINC00896 & hsa-miR-139-3p, hsa-miR-30c-2-3p \\
\hline DDX12P & hsa-miR-139-5p, \\
\hline TCAM1P & hsa-miR-139-5p, hsa-miR-221-5p, hsa-miR-30c-2-3p \\
\hline TPTEP1 & hsa-miR-142-3p, hsa-miR-708-5p \\
\hline FAM95B1 & hsa-miR-143-5p, hsa-miR-9-5p \\
\hline LINC00472 & hsa-miR-143-5p, hsa-miR-96-5p \\
\hline LINC00930 & hsa-miR-143-5p, \\
\hline RAMP2-AS1 & hsa-miR-143-5p \\
\hline CHIAP2 & hsa-miR-182-5p, hsa-miR-708-3p, \\
\hline LOC399815 & hsa-miR-30a-3p, hsa-miR-3614-5p, hsa-miR-378a-3p, hsa-miR-378c \\
\hline LRRC37A6P & hsa-miR-30a-3p, hsa-miR-338-5p, hsa-miR-378a-3p, hsa-miR-378c \\
\hline BMS1P20 & hsa-miR-30c-2-3p, hsa-miR-378a-3p, hsa-miR-378c \\
\hline LOC285629 & hsa-miR-30c-2-3p, \\
\hline MGC27382 & hsa-miR-33a-5p \\
\hline AFAP1-AS1 & hsa-miR-3614-5p, hsa-miR-378a-3p, hsa-miR-378c, hsa-miR-451a \\
\hline SNHG4 & hsa-miR-3614-5p \\
\hline UCA1 & hsa-miR-3614-5p \\
\hline CECR7 & hsa-miR-378a-3p, hsa-miR-378c \\
\hline RAET1K & hsa-miR-378a-3p, hsa-miR-378c \\
\hline PVT1 & hsa-miR-378c, \\
\hline UNQ6494 & hsa-miR-378c \\
\hline DGCR5 & hsa-miR-486-5p \\
\hline
\end{tabular}


Table III. miRNAs targeting LUAD-specific mRNAs.

\begin{tabular}{|c|c|}
\hline miRNAs & mRNAs \\
\hline hsa-miR-127-5p & ATOH8, PKNOX2, SYNPO2L \\
\hline hsa-miR-135b-5p & ADARB2, ARC, NTNG1, PCYT1B, PGR, PRIMA1, SCN2B \\
\hline hsa-miR-139-3p & SOX11 \\
\hline hsa-miR-139-5p & MEX3A, NRK, TRIM9 \\
\hline hsa-miR-143-5p & FAT3, TPPP \\
\hline hsa-miR-182-5p & $\begin{array}{l}\text { AATK, ANGPTL1, BDNF, CACNB4, DKK2, EPAS1, FAM107A, FAT3, FIGF, KCNMB2, LDB3, } \\
\text { NRN1, ST6GALNAC3, ZFP36 }\end{array}$ \\
\hline hsa-miR-210-3p & HIF3A \\
\hline hsa-miR-221-5p & CACNA1E, NAT8L, RAD51 \\
\hline hsa-miR-30a-3p & KCNE4 \\
\hline hsa-miR-30c-2-3p & INHBE, MCHR1, MNX1, NAT8L, PACSIN1 \\
\hline hsa-miR-338-5p & ONECUT2 \\
\hline hsa-miR-33a-5p & AFF3, SLC24A4 \\
\hline hsa-miR-3607-3p & CDH13, NECAB1, TNXB \\
\hline hsa-miR-3614-5p & ELAVL2, EPN3, FAM155B, GAP43, THBS2, UBE2QL1 \\
\hline hsa-miR-378a-3p & SLC2A1, SULF1 \\
\hline hsa-miR-378c & SLC2A1, SULF1 \\
\hline hsa-miR-708-3p & AFF2, AFF3, MDGA1, CNTFR, GPM6A, MASP1, MDGA1 \\
\hline hsa-miR-9-5p & CNTFR, GABRB2, NTNG1, SCN2B, SHC3, TMTC1, TSPAN18 \\
\hline hsa-miR-96-5p & $\begin{array}{l}\text { AHNAK, CACNB4, COL13A1, FREM1, HBEGF, LDB3, LRRC4, NR4A3, RIMS4, SCNN1G, } \\
\text { SLC1A1, SOX5, TLL1 }\end{array}$ \\
\hline
\end{tabular}
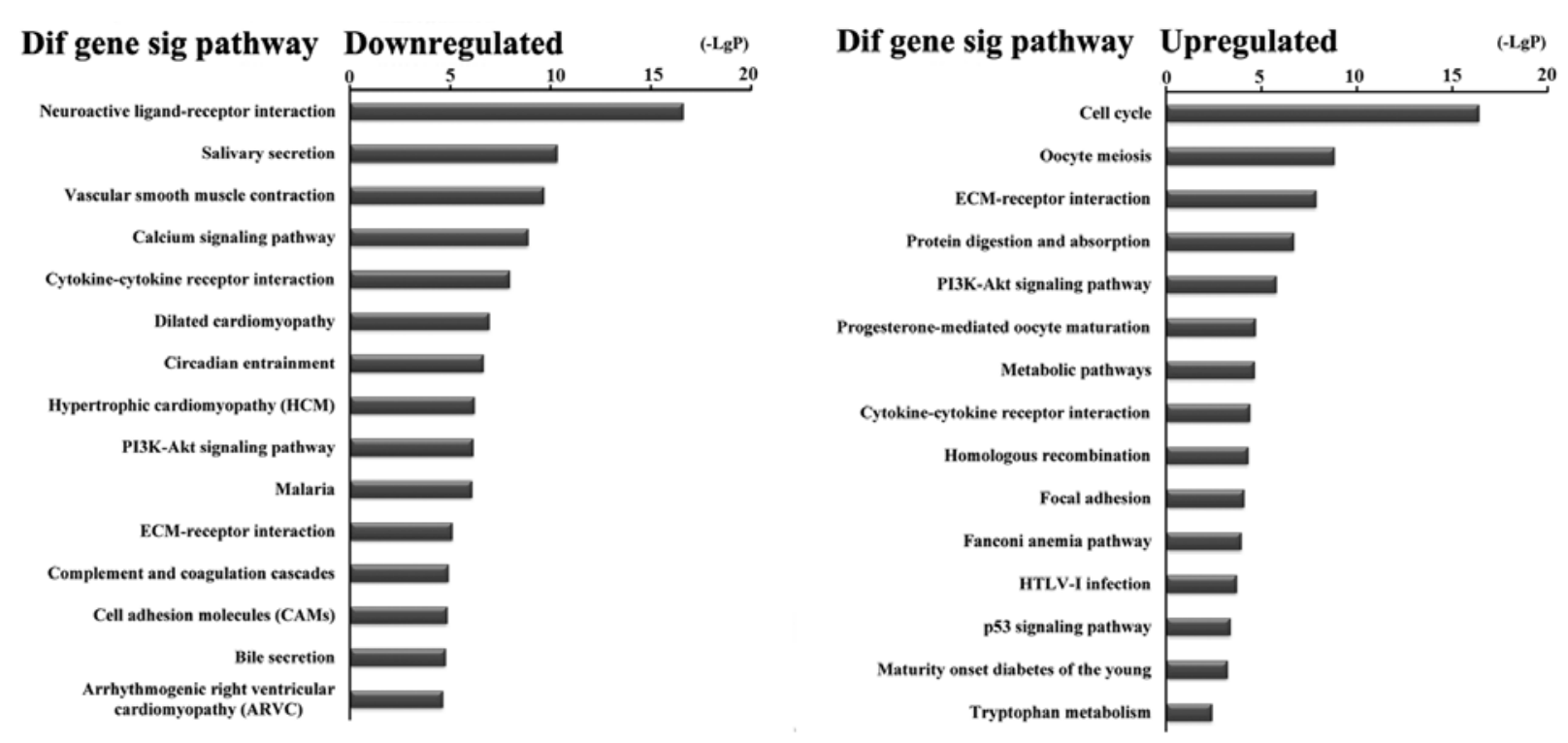

Figure 5. Top 15 enrichment of KEGG pathways for aberrantly expressed intersection mRNAs (the bar plot shows the enrichment scores of the significant top 15 enrichment KEGG pathways).

Prediction of miRNA targets and ceRNA network construction. In order to establish IncRNA-miRNA-mRNA ceRNA network, lncRNAs and mRNAs targeted by miRNAs were identified from the above data. In the present study, in total we identified 1030 miRNAs from TCGA database. A total of 118 aberrantly expressed miRNAs were found between LUAD patient tissues and adjacent non-tumor lung tissues (absolute fold change $>2, \mathrm{P}<0.05$ ). Based on comparison of the above 4 groups, 25 miRNAs were selected from the intersection aberrantly expressed miRNAs (Fig. 3C). Subsequently, we focused on the targeted relationship between these 25 miRNAs and the above 41 specific intersection lncRNAs. In the lncRNAmiRNA-mRNA ceRNA network, miRNAs interact with lncRNAs through MREs, starBase v2.0 was performed to detect the potential MREs. The results showed that 22 specific miRNAs interacted with 29 specific lncRNAs (Table II). 


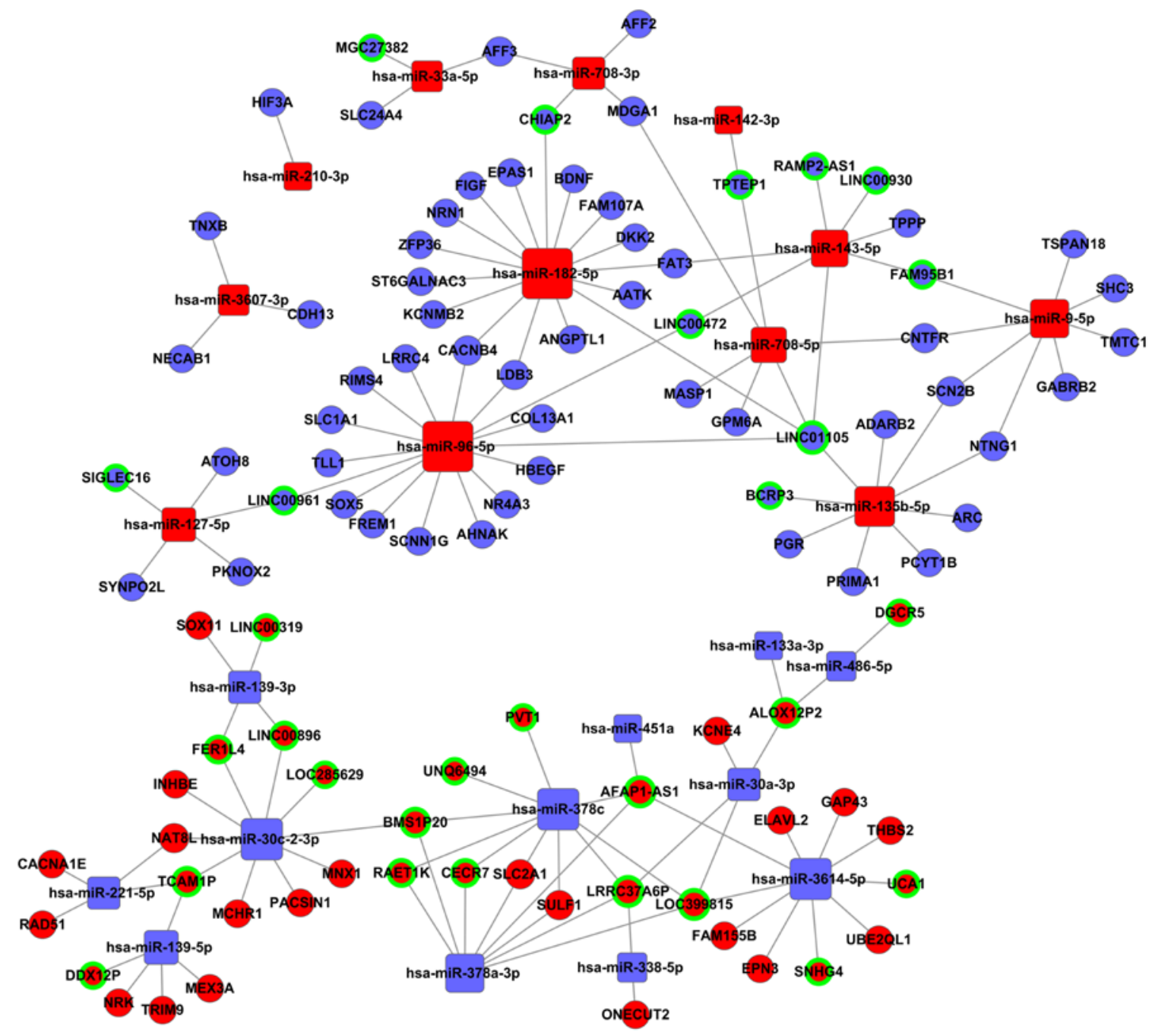

Figure 6. The lncRNA-miRNA-mRNA ceRNA network. Blue diamonds, downregulated miRNAs; blue balls, downregulated mRNAs; blue balls surrounded by green rings, downregulated lncRNAs. Red diamonds, upregulated miRNAs; red balls, upregulated mRNAs; red balls surrounded by green rings, upregulated lncRNAs.

Table IV. The correlations between LUAD specific lncRNAs from ceRNA network and clinical features.

Comparisons Downregulated Upregulated

Gender (female vs. male)

Race (White vs. Asian)

Tumor pathological stage (I-II vs. III-IV)

TNM staging system (T1 + T2 vs. T3 + T4)

Lymph node metastasis (no vs. yes)

Patient outcome assessment (dead vs. alive)
BCRP3

BCRP3, LINC01105

LINC01105, TPTEP1,

LINC00472, RAMP2-AS1

BCRP3, LINC00472, LINC00930

LINC00961, TPTEP1, LINC00472, LINC00930, MGC27382
ALOX12P2, TCAM1P, UCA1, LOC399815, SNHG4 LINC00896, SNHG4 AFAP1-AS1, SNHG4, CECR7, RAET1K, PVT1

DDX12P

FER1L4, LINC00896, BMS1P20
In the next step, mRNA targeted by miRNAs was performed. Based on the miRNAs described in Table II, TargetScan and miRTarBase were employed to predict miRNA-targeted mRNA. We identified 19 specific miRNAs related to the 72 intersection mRNAs (Table III). Some of their targets are cancer-associated genes, such as BDNF, CACNB4, DKK2, EPAS1, FIGF, RAD51, THBS2 and SLC2A1.

Based on the above data (Tables II and III), the lncRNAmiRNA-mRNA ceRNA network was constructed. The ceRNA network was plotted with Cytoscape 3.0. Fig. 6, shows 

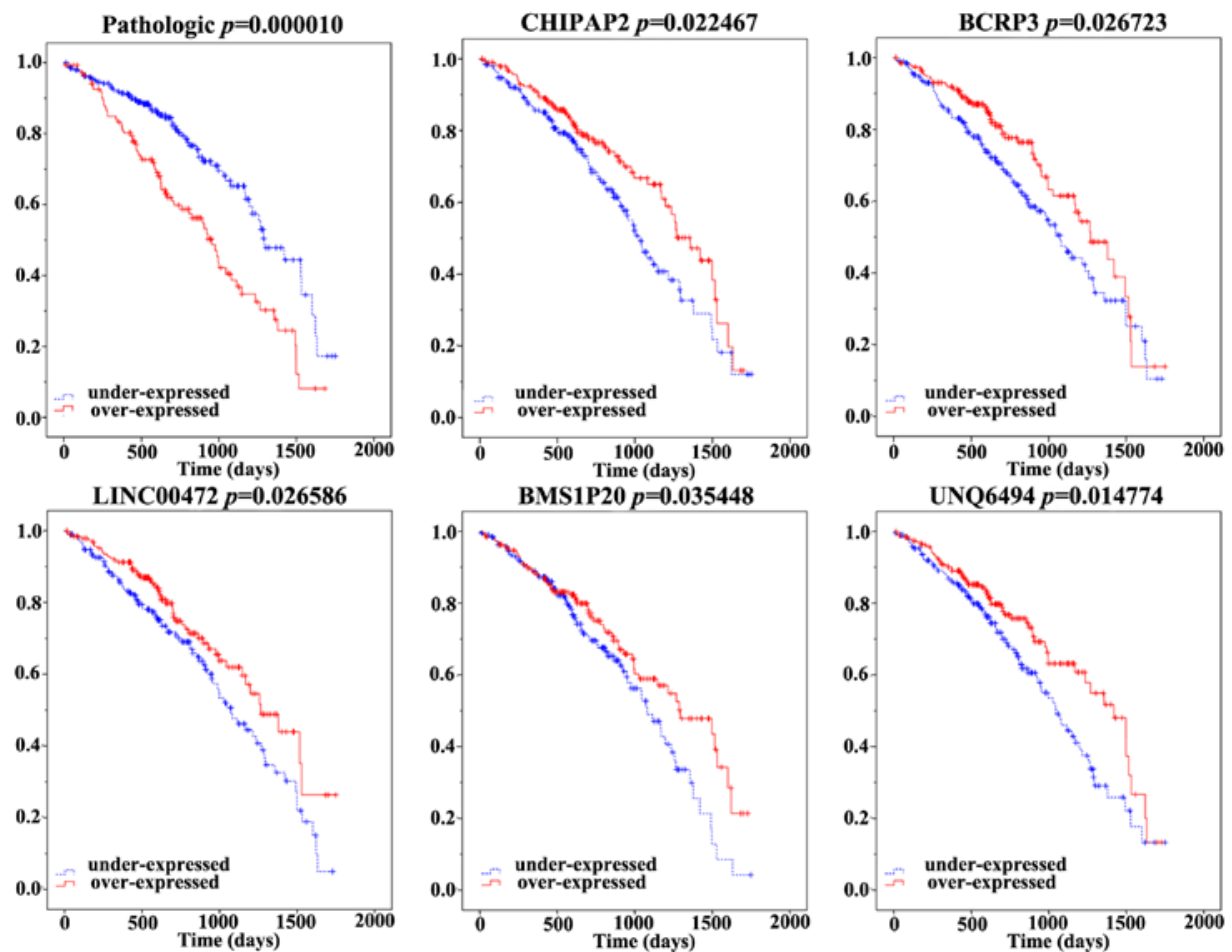

Figure 7. Kaplan-Meier survival curves for 5 lncRNAs associated with overall survival. Horizontal axis, overall survival time, days; vertical axis, survival function.

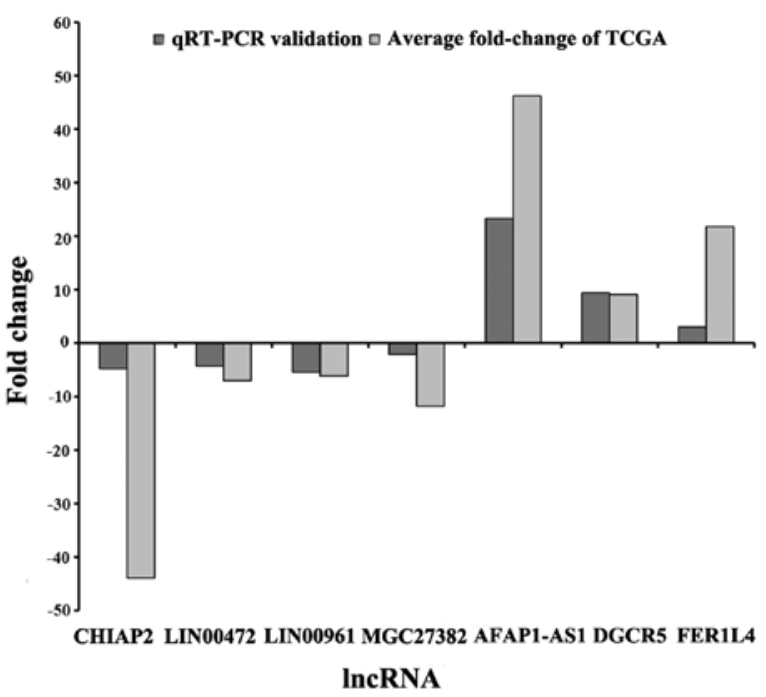

Figure 8. qRT-PCR validation of 7 differentially expressed key lncRNAs. Comparison of fold change ( $\left.2^{-\Delta \Delta C}\right)$ of 1 ncRNAs between TCGA and qRT-PCR results.

29 lncRNAs, 24 miRNAs and 72 mRNAs that are involved in the proposed ceRNA network.

The correlations between LUAD specific lncRNAs and clinical features. The 29 specific IncRNAs from the ceRNA network were further analyzed according to clinical features including gender, race, tumor pathological stage, TNM staging system, lymph node metastasis, and patient outcome assessment at diagnosis in TCGA database. There were 21 LUAD-specific lncRNAs, the expression levels of which were significantly aberrantly expressed in clinical feature comparisons (P<0.05; Table IV). Six lncRNAs (BCRP3, ALOX12P2, TCAM1P, UCA1, LOC399815 and SNHG4) were aberrantly expressed in gender, 2 lncRNAs (LINC00896 and SNHG4) were aberrantly expressed in race, 7 lncRNAs (BCRP3, LINC01105, AFAP1-AS1, SNHG4, CECR7, RAET1K and PVT1) were aberrantly expressed in tumor pathological stage, 5 lncRNAs (LINC01105, TPTEP1, LINC00472, RAMP2-AS1 and DDX12P) were aberrantly expressed in TNM staging system, 3 lncRNAs (BCRP3, LINC00472 and LINC00930) were aberrantly expressed in lymph node metastasis, and 8 lncRNAs (LINC00961, TPTEP1, LINC00472, LINC00930, MGC27382, FER1L4, LINC00896 and BMS1P20) were aberrantly expressed in patient outcome assessment.

In order to identify the 29 specific IncRNAs with prognostic characteristics, these IncRNAs in the TCGA database were profiled with the univariate Cox proportional hazards regression model and 5 specific lncRNAs were found significantly associated with OS (log-rank $\mathrm{P}<0.05)$. While the remaining 5 (BCRP3, LINC00472, CHIAP2, BMS1P20 and UNQ6494) were positively correlated with OS (Fig. 7).

$q R T-P C R$ verification. Finally, we randomly selected 7 specific lncRNAs (AFAP1-AS1, CHIAP2, DGCR5, FER1L4, LINC00472, LINC00961 and MGC27382) to validate the reliability and validity of the above analysis results. We applied the paired t-test to assess the differences between the LUAD tumor tissues and the adjacent non-tumor lung tissues. The results showed that CHIAP2, LINC00472, LINC00961 and MGC27382 were downregulated in LUAD tumor tissues when compared with adjacent non-tumor lung tissues, while AFAP1-AS1, CHIAP2 and FER1L4 were 
Table V. Relative expression of 7 lncRNAs in the LUAD tumor tissues and the adjacent non-tumor lung tissues.

\begin{tabular}{|c|c|c|c|c|c|}
\hline lncRNAs & Tissues & $\Delta \mathrm{C}_{\mathrm{T}}($ mean $\pm \mathrm{SD})$ & $\Delta \Delta \mathrm{C}_{\mathrm{T}}($ mean $\pm \mathrm{SD})$ & $2^{-\Delta \Delta C t}$ & P-value ${ }^{a}$ \\
\hline CHIAP2 & $\begin{array}{l}\mathrm{C} \\
\mathrm{N}\end{array}$ & $\begin{array}{c}11.492 \pm 3.274 \\
9.225 \pm 2.448\end{array}$ & $2.267 \pm 3.834$ & 0.206 & $0.000^{\mathrm{b}}$ \\
\hline LINC00472 & $\begin{array}{l}\mathrm{C} \\
\mathrm{N}\end{array}$ & $\begin{array}{l}10.463 \pm 2.038 \\
10.188 \pm 3.091\end{array}$ & $0.275 \pm 3.143$ & 0.826 & $0.002^{\mathrm{b}}$ \\
\hline LINC00961 & $\begin{array}{l}\mathrm{C} \\
\mathrm{N}\end{array}$ & $\begin{array}{c}11.361 \pm 2.027 \\
9.253 \pm 2.372\end{array}$ & $2.109 \pm 2.917$ & 0.232 & $0.000^{\mathrm{b}}$ \\
\hline MGC27382 & $\begin{array}{l}\mathrm{C} \\
\mathrm{N}\end{array}$ & $\begin{array}{l}8.419 \pm 2.559 \\
5.977 \pm 2.397\end{array}$ & $2.443 \pm 3.059$ & 0.184 & $0.000^{\mathrm{b}}$ \\
\hline AFAP1-AS1 & $\begin{array}{l}\mathrm{C} \\
\mathrm{N}\end{array}$ & $\begin{array}{c}9.143 \pm 3.779 \\
13.682 \pm 4.450\end{array}$ & $-4.539 \pm 4.813$ & 23.244 & $0.000^{\mathrm{b}}$ \\
\hline DGCR5 & $\begin{array}{l}\mathrm{C} \\
\mathrm{N}\end{array}$ & $\begin{array}{l}13.504 \pm 2.427 \\
16.669 \pm 3.591\end{array}$ & $-3.227 \pm 4.183$ & 9.365 & $0.000^{\mathrm{b}}$ \\
\hline FER1L4 & $\begin{array}{l}\mathrm{C} \\
\mathrm{N}\end{array}$ & $\begin{array}{l}7.887 \pm 1.983 \\
9.472 \pm 1.994\end{array}$ & $-1.585 \pm 2.840$ & 2.999 & $0.000^{\mathrm{b}}$ \\
\hline
\end{tabular}

${ }^{\text {a }} \mathrm{P}$-value, $\Delta \Delta \mathrm{C}$ T of LUAD tumor tissues compared with adjacent non-tumor lung tissues. ${ }^{\mathrm{b}} \mathrm{P}<0.01$.
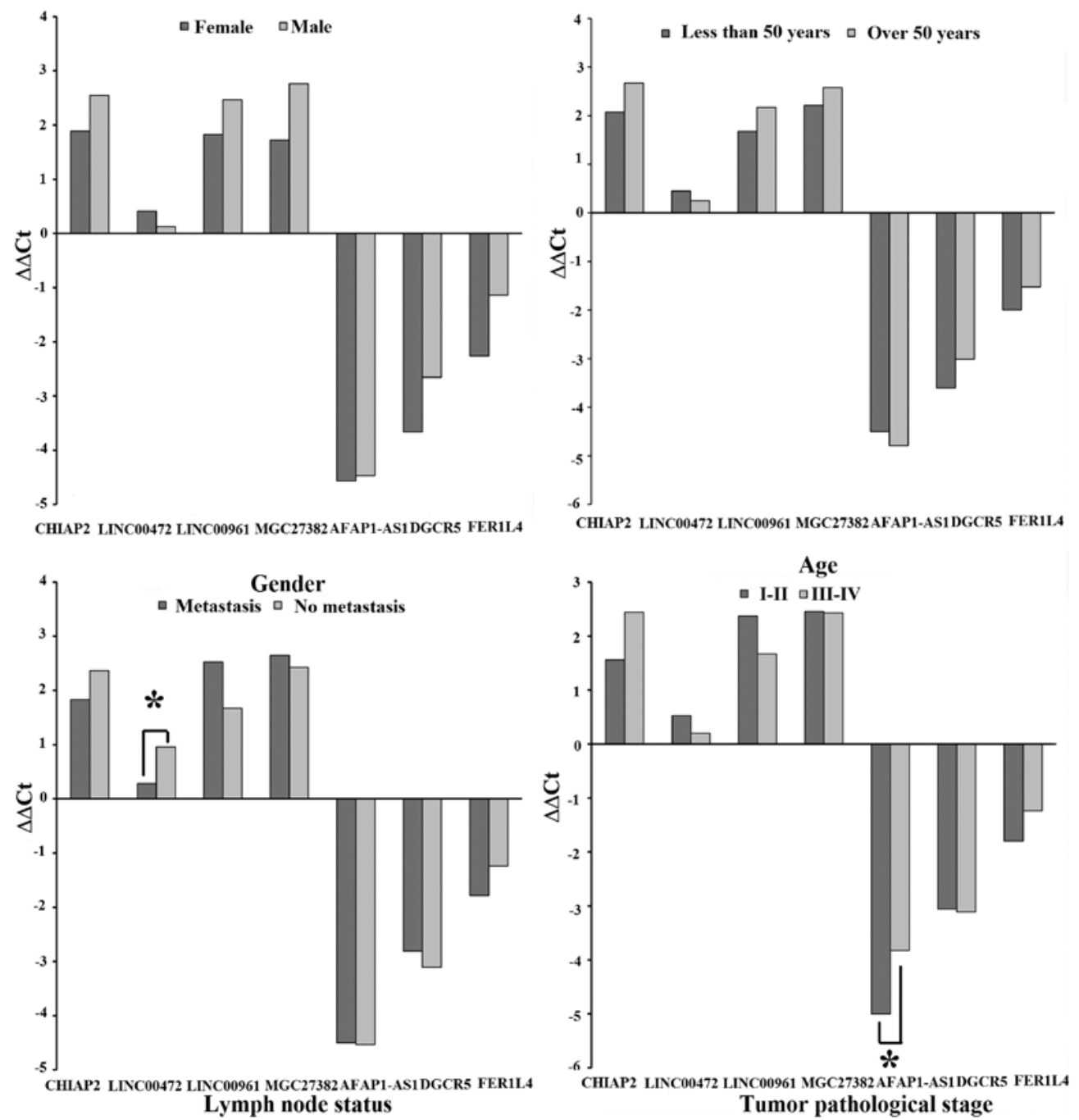

Figure 9. Association between the $\Delta \Delta$ CT of AFAP1-AS1, CHIAP2, DGCR5, FER1L4, LINC00472, LINC00961 and MGC27382 and clinicopathological characteristics in 53 LUAD $\left({ }^{*} \mathrm{P}<0.05\right)$. 
upregulated in LUAD tumor tissues (Fig. 8 and Table V). The results from the qRT-PCR validation in 53 newly diagnosed LUAD patients were consistent with the above bioinformatics results (Table I). Then, we analyzed the 7 lncRNAs with clinical features. The result showed that AFAP1-AS1 was significantly related with tumor pathological stage, and LINC00472 was significantly related with lymph node metastasis (Fig. 9). The clinical relevance of the 2 lncRNAs was substantially identical with the results of bioinformatics analysis. The above results revealed that our bioinformatics analysis was credible.

\section{Discussion}

Lung cancer is current the neoplasia with the highest global incidence and mortality $(36,37)$. LUAD, a cancer that originates in lung glandular cells, is the most frequent type of lung cancer (38). It has been reported that LUAD is steadily rising in both men and women replacing lung squamous cell carcinoma as the most common pathological type of lung cancer in Korea (39). While major improvements in diagnosis, surgical skills, and medical treatments of lung cancer have been applied over the past few years, the average 5-year survival rate remains $\sim 10 \%$ (40), mainly because diagnosis cannot find all early stage of the disease. Although several investigative methods are available to support diagnosis and staging of LUAD, such as biopsy sampling, mediastinoscopy, and bronchoscopy, these methods are time-consuming, which may lead to delays in early treatment (41). Therefore, to improve this situation, the identification of cancer-related genes and the exact regulatory mechanism of LUAD initiation and development have received increasing attention.

Aberrant expression of lncRNAs has been widely observed in various diseases, and studies have shown that the dysregulated lncRNAs emerge as key roles in vital biological functions of cancers $(42,43)$. However, only a few studies have described IncRNA profiles in LUAD by microarray analysis (31). Song et al (44) have constructed IncRNA-mRNA network by dysregulated IncRNAs and mRNAs based on ceRNA hypothesis. To date, only a few studies represent the interaction between IncRNA and mRNA, or lncRNA and miRNA in LUAD, the results of which showed that lncRNAs can function as a key part of ceRNA network, but such ceRNA network is still poorly described (45-48). The ceRNA hypothesis has been proposed as a novel regulatory mechanism functioning through miRNA competition $(27,49)$. With further research of ceRNA crosstalk, studies showed that ceRNA genes were mediated by miRNAs, which interacted with IncRNAs, in the complex ceRNA network (50).

In the present study, we identified IncRNAs, miRNAs and mRNAs from TCGA database, which were related with TNM stage and lymph node metastasis in LUAD. Then, we constructed the IncRNA-miRNA-mRNA ceRNA network with TNM stage and lymph node metastasis-related LUAD-specific lncRNAs, miRNAs and mRNAs, which provides integrated biological views of the ceRNA network. The constructed LUAD-related lncRNA-associated ceRNA network provides important hints for detecting the key RNAs of ceRNA-mediated gene regulatory network in the initiation and development of LUAD. Next, we selected the cancer specific key lncRNAs from ceRNA network and investigated their distributions in different LUAD clinical features and their associations with overall survival on the basis of RNA sequencing profile of 465 LUAD tissues and 49 adjacent non-tumor lung tissues. We randomly selected 7 specific lncRNAs (AFAP1-AS1, CHIAP2, DGCR5, FER1L4, LINC00472, LINC00961 and MGC27382) and analyzed their expression levels in the 53 newly diagnosed tumor tissues of LUAD patients and adjacent non-tumor lung tissues using qRT-PCR.

Based on the RNA sequence data from TCGA database, we divided LUAD patients into 4 groups with TNM stage and lymph node metastasis as condition, and they were compared with adjacent non-tumor lung tissues, respectively. Then, we selected the intersection RNAs. In the next step, we investigated bioinformatics of aberrantly expressed mRNAs, and the front enriched functional annotation of GO and KEGG pathway might be the potential function of lncRNA. The results of GO biological process terms showed that the aberrantly expressed genes mainly concentrate on cellular functions, metabolism and immune functions. Based on KEGG pathway analysis, a few cancer-related pathways were detected, including p53 signaling pathway, MAPK signaling pathway and Wnt signaling pathway. Wu et al (51) merged two datasets to find novel target genes and pathways to explain the pathogenicity of LUAD, and they found that PPM1D and GADD45B may regulate LUAD progression through p53 signaling pathway. Wang et al (52) established a co-culture system of A549 cells and bone marrow-derived cells (HS-5) to investigate the molecular mechanism of BMP9 in LUAD and the bone metastatic microenvironment. Then, he found BMP9 can inhibit the growth and migration of LUAD A549 cells through the MAPK/ERK and $\mathrm{NF}-\kappa \mathrm{B}$ pathways. It was also reported that Wnt signaling pathways were related with LUAD (53-55).

Growing body of evidence has presented that lncRNAs function as a crucial component of ceRNA network by modulating other RNA transcripts (19,30,33,56-58). For example, HOTAIR may act as an endogenous sink by binding miR-331-3p, thereby abolishing the miRNA-induced inhibitory activity on the HER2 3'-UTR and increasing an additional level of post-transcriptional regulation (59). Hence, the potential connection lncRNA, miRNA and mRNA may exist in initiation and development of LUAD. In the present study, we constructed the lncRNA-miRNA-mRNA ceRNA network to reveal a novel ceRNA regulatory network in LUAD. We found some cancer special lncRNAs, such as FER1L4 (60), MGC27382 (61), UCA1 (25), AFAP1-AS1 (62), were also reported in cancers acting as potential diagnosis and prognostic biomarkers. In addition, we found THBS2 (63), RAD51 (64), SLC2A1 (65), BDNF (66), in the ceRNA network were also related with initiation and development of cancers. In the present study, we analyzed the LUAD-specific IncRNA indirectly related mRNA signal pathways involved in ceRNA network. The results of pathway analysis showed that there were 9 pathways related with cancer. Therefore, our results suggested that the key lncRNAs may play an important role in initiation and development of LUAD and the cancer genes related pathways.

With respect to the associations between 29 cancer specific lncRNAs from ceRNA network and clinical features, including 
gender, tumor pathological stage, TNM staging system, lymph node metastasis and patient outcome assessment, we found that 21 lncRNAs were related to clinical features. Eight of these 21 lncRNAs were reported to be associated with cancer. For example, UCA1 was reported to be an indicator of early gastric cancer (67). However, there is no report on the association between LUAD and the above IncRNAs features. Then we analyzed the associations between 29 cancer-specific lncRNAs and the patient survival, and we found that 5 lncRNAs were related to LUAD OS.

Finally, 7 cancer specific lncRNAs were randomly selected to verify the expressions of specific lncRNAs and credibility of bioinformatics analysis using qRT-PCR. The expression data from TCGA and verification result of 53 newly diagnosed LUAD patients were $100 \%$ in agreement. Then, we performed the correlation analysis between the 7 cancer specific lncRNAs and clinical features. The results showed that AFAP1-AS1 and LINC00472 were significantly correlated with tumor pathological stage and lymph node metastasis, respectively. These above results revealed that our bioinformatics analysis is credible.

Based on these above LUAD OS results, only 1 of these 5 LUAD-OS related lncRNAs (LINC00472) was aberrantly expressed in clinical feature. Therefore, IncRNAs that aberrantly expressed in clinical feature comparisons may not be necessarily related with OS (29). Among these 5 lncRNAs only LINC00472 has been reported in the survival of carcinoma and the other lncRNAs (BCRP3, CHIAP2, BMS1P20 and UNQ6494) have not been reported (68). Furthermore, a recent study also found that high LINC00472 expression could significantly reduce risk of relapse and death in breast cancer patients, and LINC00472 could also suppress breast cancer cell proliferation and migration (69). It implies that LINC00472 may play an important role in LUAD.

It has been reported that upregulated AFAP1-AS1 was associated with the poor prognosis of non-small cell lung cancer (NSCLC) patients (70). Furthermore, AFAP1-AS1 was also reported to be associated with prognosis, cell proliferation and invasion of other cancers $(62,71,72)$. Combined with the present study (Tables I, IV, V and Figs. 6-8), showing that the expression of AFAP1-AS1 and LINC00472 were dysregulated in LUAD from TCGA database and qRT-PCR verification, and they were related with tumor pathological stage, and lymph node metastasis and OS, respectively, it reveals that AFAP1-AS1 and LINC00472 may be potential biomarkers in LUAD.

In the present study, we identified the LUAD-specific IncRNAs from hundreds of candidate lncRNAs detected from large scale samples in TCGA database, and revealed aberrant expression profiles of cancer specific IncRNAs under different clinical features. The aberrantly expressed key lncRNAs, which were identified in LUAD, may shed light on sensitive biomarkers in LUAD. Importantly, we have constructed the IncRNA-miRNA-mRNA ceRNA network to clarify the unknown ceRNA regulatory network in LUAD. In addition, qRT-PCR verification was used for the reliability and validity of expression of key lncRNAs and bioinformatics analysis. Our findings provide novel insight into better understanding of IncRNA-related ceRNA network in LUAD and potential biomarkers for diagnosis and prognosis.

\section{Acknowledgements}

The present study was supported by the National Natural Science Foundation of China $(81472939,81172618$ and 81673132), the Qing Lan Project, the 333 Project of Jiangsu Province, the Liu Da Ren Cai Gao Feng Project of Jiangsu Province and the Fundamental Research Funds for the central universities and innovative Research Project for postgraduates in Colleges of Jiangsu Province. We thank Mr. Donglin Cheng for his technical assistance.

\section{References}

1. Torre LA, Bray F, Siegel RL, Ferlay J, Lortet-Tieulent J and Jemal A: Global cancer statistics, 2012. CA Cancer J Clin 65: 87-108, 2015.

2. Jemal A, Bray F, Center MM, Ferlay J, Ward E and Forman D: Global cancer statistics. CA Cancer J Clin 61: 69-90, 2011.

3. Liu L, Wu S, Yang Y, Cai J, Zhu X, Wu J, Li M and Guan H: SOSTDC1 is down-regulated in non-small cell lung cancer and contributes to cancer cell proliferation. Cell Biosci 6: 24, 2016.

4. Li X, Shi Y, Yin Z, Xue X and Zhou B: An eight-miRNA signature as a potential biomarker for predicting survival in lung adenocarcinoma. J Transl Med 12: 159, 2014.

5. Ferlay J, Shin HR, Bray F, Forman D, Mathers C and Parkin DM: Estimates of worldwide burden of cancer in 2008: GLOBOCAN 2008. Int J Cancer 127: 2893-2917, 2010.

6. Wang Y, Zhao H, Gao X, Wei F, Zhang X, Su Y, Wang C, Li H and Ren $X$ : Identification of a three-miRNA signature as a blood-borne diagnostic marker for early diagnosis of lung adenocarcinoma. Oncotarget 7: 26070-26086, 2016.

7. Schneider F, Derrick V, Davison JM, Strollo D, Incharoen P and Dacic S: Morphological and molecular approach to synchronous non-small cell lung carcinomas: impact on staging. Mod Pathol 29: 735-742, 2016.

8. Qu J, Li M, An J, Zhao B, Zhong W, Gu Q, Cao L, Yang H and Hu C: MicroRNA-33b inhibits lung adenocarcinoma cell growth, invasion, and epithelial-mesenchymal transition by suppressing Wnt/ $\beta$-catenin/ZEB1 signaling. Int J Oncol 47: 2141-2152, 2015.

9. Murayama T, Nakaoku T, Enari M, Nishimura T, Tominaga K, Nakata A, Tojo A, Sugano S, Kohno T and Gotoh N: Oncogenic fusion gene CD74-NRG1 confers cancer stem cell-like properties in lung cancer through a IGF2 autocrine/paracrine circuit. Cancer Res 76: 974-983, 2016.

10. Wang G, Chen H and Liu J: The long noncoding RNA LINC01207 promotes proliferation of lung adenocarcinoma. Am J Cancer Res 5: 3162-3173, 2015.

11. Sana J, Faltejskova P, Svoboda M and Slaby O: Novel classes of non-coding RNAs and cancer. J Transl Med 10: 103, 2012.

12. Takahashi K, Yan I, Haga $\mathrm{H}$ and Patel T: Long noncoding RNA in liver diseases. Hepatology 60: 744-753, 2014.

13. Braconi C, Valeri N, Kogure T, Gasparini P, Huang N, Nuovo GJ, Terracciano L, Croce CM and Patel T: Expression and functional role of a transcribed noncoding RNA with an ultraconserved element in hepatocellular carcinoma. Proc Natl Acad Sci USA 108: 786-791, 2011.

14. Jiang F, Zhou X and Huang J: Long non-coding RNA-ROR mediates the reprogramming in cardiac hypertrophy. PLoS One 11: $\mathrm{e} 0152767,2016$.

15. Chang L, Qi H, Xiao Y, Li C, Wang Y, Guo T, Liu Z and Liu Q: Integrated analysis of noncoding RNAs and mRNAs reveals their potential roles in the biological activities of the growth hormone receptor. Growth Horm IGF Res 29: 11-20, 2016.

16. Voellenkle C, Garcia-Manteiga JM, Pedrotti S, Perfetti A, De Toma I, Da Silva D, Maimone B, Greco S, Fasanaro P, Creo P, et al: Implication of long noncoding RNAs in the endothelial cell response to hypoxia revealed by RNA-sequencing. Sci Rep 6: 24141, 2016.

17. Mercer TR and Mattick JS: Structure and function of long noncoding RNAs in epigenetic regulation. Nat Struct Mol Biol 20: 300-307, 2013.

18. Yoon JH, Abdelmohsen K and Gorospe M: Posttranscriptional gene regulation by long noncoding RNA. J Mol Biol 425: 3723-3730, 2013 
19. Zhou M, Wang X, Shi H, Cheng L, Wang Z, Zhao H, Yang L and Sun J: Characterization of long non-coding RNA-associated ceRNA network to reveal potential prognostic lncRNA biomarkers in human ovarian cancer. Oncotarget 7: 12598-12611, 2016.

20. Hung $\mathrm{T}$ and Chang HY: Long noncoding RNA in genome regulation: Prospects and mechanisms. RNA Biol 7: 582-585, 2010

21. Shi X, Ma C, Zhu Q, Yuan D, Sun M, Gu X, Wu G, Lv T and Song Y: Upregulation of long intergenic noncoding RNA 00673 promotes tumor proliferation via LSD1 interaction and repression of NCALD in non-small-cell lung cancer. Oncotarget 7: 25558-25575, 2016.

22. Wang PL, Liu B, Xia Y, Pan CF, Ma T and Chen YJ: Long noncoding RNA-low expression in tumor inhibits the invasion and metastasis of esophageal squamous cell carcinoma by regulating p53 expression. Mol Med Rep 13: 3074-3082, 2016.

23. Shang C, Guo Y, Zhang J and Huang B: Silence of long noncoding RNA UCA1 inhibits malignant proliferation and chemotherapy resistance to adriamycin in gastric cancer. Cancer Chemother Pharmacol 77: 1061-1067, 2016.

24. Zhang D, Cao C, Liu L and Wu D: Up-regulation of LncRNA SNHG20 predicts poor prognosis in hepatocellular carcinoma. J Cancer 7: 608-617, 2016.

25. Bian Z, Jin L, Zhang J, Yin Y, Quan C, Hu Y, Feng Y, Liu H, Fei B, Mao Y, et al: LncRNA-UCA1 enhances cell proliferation and 5-fluorouracil resistance in colorectal cancer by inhibiting miR-204-5p. Sci Rep 6: 23892, 2016.

26. Sun R, Qin C, Jiang B, Fang S, Pan X, Peng L, Liu Z, Li W, Li Y and Li G: Down-regulation of MALAT1 inhibits cervical cancer cell invasion and metastasis by inhibition of epithelialmesenchymal transition. Mol Biosyst 12: 952-962, 2016.

27. Salmena L, Poliseno L, Tay Y, Kats L and Pandolfi PP: A ceRNA hypothesis: The Rosetta Stone of a hidden RNA language? Cell 146: 353-358, 2011.

28. Pilyugin $\mathrm{M}$ and Irminger-Finger I: Long non-coding RNA and microRNAs might act in regulating the expression of BARD1 mRNAs. Int J Biochem Cell Biol 54: 356-367, 2014.

29. Zhang J, Fan D, Jian Z, Chen GG and Lai PB: Cancer specific long noncoding RNAs show differential expression patterns and competing endogenous RNA potential in hepatocellular carcinoma. PLoS One 10: e0141042, 2015.

30. Li CY, Liang GY, Yao WZ, Sui J, Shen X, Zhang YQ, Peng H, Hong WW, Ye YC, Zhang ZY, et al: Integrated analysis of long non-coding RNA competing interactions reveals the potential role in progression of human gastric cancer. Int $\mathrm{J}$ Oncol 48 1965-1976, 2016.

31. Chen J, Hu L, Chen J, Pan Q, Ding H, Xu G, Zhu P, Wen X, Huang K and Wang Y: Detection and analysis of Wnt pathway related lncRNAs expression profile in lung adenocarcinoma. Pathol Oncol Res 22: 609-615, 2016

32. McCarter JP, Mitreva MD, Martin J, Dante M, Wylie T, Rao U, Pape D, Bowers Y, Theising B, Murphy CV, et al: Analysis and functional classification of transcripts from the nematode Meloidogyne incognita. Genome Biol 4: R26, 2003.

33. Guo LL, Song CH, Wang P, Dai LP, Zhang JY and Wang KJ: Competing endogenous RNA networks and gastric cancer. World J Gastroenterol 21: 11680-11687, 2015.

34. Li JH, Liu S, Zhou H, Qu LH and Yang JH: starBase v2.0 Decoding miRNA-ceRNA, miRNA-ncRNA and protein-RNA interaction networks from large-scale CLIP-Seq data. Nucleic Acids Res 42 (D1): D92-D97, 2014.

35. Hsu SD, Tseng YT, Shrestha S, Lin YL, Khaleel A, Chou CH Chu CF, Huang HY, Lin CM, Ho SY, et al: miRTarBase update 2014: An information resource for experimentally validated miRNA-target interactions. Nucleic Acids Res 42 (D1): D78-D85, 2014.

36. Siegel R, Naishadham D and Jemal A: Cancer statistics, 2012 CA Cancer J Clin 62: 10-29, 2012.

37. Meza R, Meernik C, Jeon J and Cote ML: Lung cancer incidence trends by gender, race and histology in the United States, 19732010. PLoS One 10: e0121323, 2015.

38. Kerr KM: Pulmonary adenocarcinomas: Classification and reporting. Histopathology 54: 12-27, 2009.

39. Park JY and Jang SH: Epidemiology of lung cancer in Korea: Recent trends. Tuberc Respir Dis (Seoul) 79: 58-69, 2016.

40. Ortea I, Rodríguez-Ariza A, Chicano-Gálvez E, Arenas Vacas MS and Jurado Gámez B: Discovery of potential protein biomarkers of lung adenocarcinoma in bronchoalveolar lavage fluid by SWATH MS data-independent acquisition and targeted data extraction. J Proteomics 138: 106-114, 2016.
41. Navani N, Nankivell M, Lawrence DR, Lock S, Makker H, Baldwin DR, Stephens RJ, Parmar MK, Spiro SG, Morris S, et al; Lung-BOOST trial investigators: Lung cancer diagnosis and staging with endobronchial ultrasound-guided transbronchial needle aspiration compared with conventional approaches: An open-label, pragmatic, randomised controlled trial. Lancet Respir Med 3: 282-289, 2015.

42. Esteller M: Non-coding RNAs in human disease. Nat Rev Genet 12: 861-874, 2011

43. Harries LW: Long non-coding RNAs and human disease. Biochem Soc Trans 40: 902-906, 2012.

44. Song C, Zhang J, Liu Y, Pan H, Qi HP, Cao YG, Zhao JM, Li S, Guo J, Sun HL, et al: Construction and analysis of cardiac hypertrophy-associated lncRNA-mRNA network based on competitive endogenous RNA reveal functional lncRNAs in cardiac hypertrophy. Oncotarget 7: 10827-10840, 2016.

45. Peters T, Hermans-Beijnsberger S, Beqqali A, Bitsch N, Nakagawa S, Prasanth KV, de Windt LJ, van Oort RJ, Heymans S and Schroen B: Long non-coding RNA malat-1 is dispensable during pressure overload-induced cardiac remodeling and failure in mice. PLoS One 11: e0150236, 2016.

46. Zhou KI, Parisien M, Dai Q, Liu N, Diatchenko L, Sachleben JR and Pan T: $\mathrm{N}^{6}$-Methyladenosine modification in a long noncoding RNA hairpin predisposes its conformation to protein binding. J Mol Biol 428 (5 Pt A): 822-833, 2016.

47. Wei G: Bioinformatics analysis of microRNA comprehensive regulatory network in congenital microtia. Int J Pediatr Otorhinolaryngol 79: 1727-1731, 2015.

48. Liu S, Song L, Zeng S and Zhang L: MALAT1-miR-124-RBG2 axis is involved in growth and invasion of HR-HPV-positive cervical cancer cells. Tumour Biol 37: 633-640, 2016.

49. Tay Y, Rinn J and Pandolfi PP: The multilayered complexity of ceRNA crosstalk and competition. Nature 505: 344-352, 2014.

50. Karreth FA and Pandolfi PP: ceRNA cross-talk in cancer: When ce-bling rivalries go awry. Cancer Discov 3: 1113-1121, 2013.

51. Wu X, Zang W, Cui S and Wang M: Bioinformatics analysis of two microarray gene-expression data sets to select lung adenocarcinoma marker genes. Eur Rev Med Pharmacol Sci 16: $1582-1587,2012$

52. Wang J, Weng Y, Zhang M, Li Y, Fan M, Guo Y, Sun Y, Li W and Shi Q: BMP9 inhibits the growth and migration of lung adenocarcinoma A549 cells in a bone marrow stromal cell-derived microenvironment through the MAPK/ERK and $N F-\kappa B$ pathways. Oncol Rep 36: 410-418, 2016.

53. Sunaga N, Kohno T, Kolligs FT, Fearon ER, Saito R and Yokota J: Constitutive activation of the Wnt signaling pathway by CTNNB1 (beta-catenin) mutations in a subset of human lung adenocarcinoma. Genes Chromosomes Cancer 30: 316-321, 2001.

54. Jiang HL, Jiang LM and Han WD: Wnt/beta-catenin signaling pathway in lung cancer stem cells is a potential target for the development of novel anticancer drugs. J BUON 20: 1094-1100, 2015.

55. Qi W, Chen J, Cheng X, Huang J, Xiang T, Li Q, Long H and Zhu B: Targeting the Wnt-regulatory protein CTNNBIP1 by microRNA-214 enhances the stemness and self-renewal of cancer stem-like cells in lung adenocarcinomas. Stem Cells 33: 3423-3436, 2015

56. Hu Y, Tian H, Xu J and Fang JY: Roles of competing endogenous RNAs in gastric cancer. Brief Funct Genomics 15: 266-273, 2015.

57. Wu Q, Guo L, Jiang F, Li L, Li Z and Chen F: Analysis of the miRNA-mRNA-lncRNA networks in $\mathrm{ER}^{+}$and $\mathrm{ER}^{-}$breast cancer cell lines. J Cell Mol Med 19: 2874-2887, 2015.

58. Song X, Cao G, Jing L, Lin S, Wang X, Zhang J, Wang M, Liu W and Lv C: Analysing the relationship between lncRNA and protein-coding gene and the role of IncRNA as ceRNA in pulmonary fibrosis. J Cell Mol Med 18: 991-1003, 2014.

59. Liu XH, Sun M, Nie FQ, Ge YB, Zhang EB, Yin DD, Kong R, Xia R, Lu KH, Li JH, et al: Lnc RNA HOTAIR functions as a competing endogenous RNA to regulate HER 2 expression by sponging miR-331-3p in gastric cancer. Mol Cancer 13: 92, 2014.

60. Liu Z, Shao Y, Tan L, Shi H, Chen S and Guo J: Clinical significance of the low expression of FER1L4 in gastric cancer patients. Tumour Biol 35: 9613-9617, 2014.

61. Kim ST, Sohn I, Do IG, Jang J, Kim SH, Jung IH, Park JO, Park YS, Talasaz A, Lee J, et al: Transcriptome analysis of CD133-positive stem cells and prognostic value of survivin in colorectal cancer. Cancer Genomics Proteomics 11: 259-266, 2014. 
62. Zhang JY, Weng MZ, Song FB, Xu YG, Liu Q, Wu JY, Qin J, Jin T and Xu JM: Long noncoding RNA AFAP1-AS1 indicates a poor prognosis of hepatocellular carcinoma and promotes cell proliferation and invasion via upregulation of the RhoA/Rac2 signaling. Int J Oncol 48: 1590-1598, 2016.

63. Tian ZQ, Li ZH, Wen SW, Zhang YF, Li Y, Cheng JG and Wang GY: Identification of commonly dysregulated genes in non-small-cell lung cancer by integrated analysis of microarray data and qRT-PCR validation. Lung 193: 583-592, 2015.

64. Jiang Y, Verbiest T, Devery AM, Bokobza SM, Weber AM, Leszczynska KB, Hammond EM and Ryan AJ: Hypoxia potentiates the radiation-sensitizing effect of olaparib in human non-small cell lung cancer xenografts by contextual synthetic lethality. Int J Radiat Oncol Biol Phys 95: 772-781, 2016.

65. Tang Y, Cao K, Wang Q, Chen J, Liu R, Wang S, Zhou J and $\mathrm{Xie} \mathrm{H}$ : Silencing of CerS6 increases the invasion and glycolysis of melanoma WM35, WM451 and SK28 cell lines via increased GLUT1-induced downregulation of WNT5A. Oncol Rep 35: 2907-2915, 2016

66. Correa DD, Satagopan J, Cheung K, Arora AK, KryzaLacombe M, Xu Y, Karimi S, Lyo J, DeAngelis LM and Orlow I: COMT, BDNF, and DTNBP1 polymorphisms and cognitive functions in patients with brain tumors. Neuro Oncol: Apr 18, 2016. pii: now057 (Epub ahead of print).
67. Gao J, Cao R and Mu H: Long non-coding RNA UCA1 may be a novel diagnostic and predictive biomarker in plasma for early gastric cancer. Int J Clin Exp Pathol 8: 12936-12942, 2015.

68. Shen Y, Wang Z, Loo LW, Ni Y, Jia W, Fei P, Risch HA, Katsaros D and Yu H: LINC00472 expression is regulated by promoter methylation and associated with disease-free survival in patients with grade 2 breast cancer. Breast Cancer Res Treat 154: 473-482, 2015.

69. Shen Y, Katsaros D, Loo LW, Hernandez BY, Chong C, Canuto EM, Biglia N, Lu L, Risch H, Chu WM, et al: Prognostic and predictive values of long non-coding RNA LINC00472 in breast cancer. Oncotarget 6: 8579-8592, 2015.

70. Deng J, Liang Y, Liu C, He S and Wang S: The up-regulation of long non-coding RNA AFAP1-AS1 is associated with the poor prognosis of NSCLC patients. Biomed Pharmacother 75: 8-11, 2015.

71. Lu X, Zhou C, Li R, Liang Z, Zhai W, Zhao L and Zhang S: Critical role for the long non-coding RNA AFAP1-AS1 in the proliferation and metastasis of hepatocellular carcinoma. Tumour Biol 7: 9699-9707, 2016.

72. Yang F, Lyu S, Dong S, Liu Y, Zhang X and Wang O: Expression profile analysis of long noncoding RNA in HER-2-enriched subtype breast cancer by next-generation sequencing and bioinformatics. Onco Targets Ther 9: 761-772, 2016. 